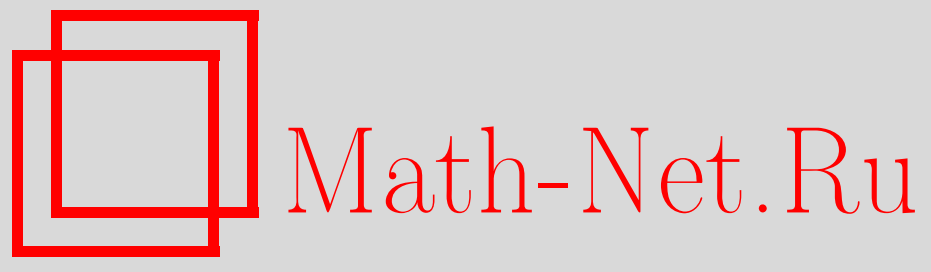

В. А. Белавин, Рекуррентные соотношения для $N=1$ суперсимметричных конформных блоков, ТМФ, 2007, том 152, номер 3, 476-487

DOI: https://doi.org/10.4213/tmf6104

Использование Общероссийского математического портала Math-Net.Ru подразумевает, что вы прочитали и согласны с пользовательским соглашением http://www . mathnet.ru/rus/agreement

Параметры загрузки:

IP : 3.85 .73 .92

26 апреля 2023 г., $07: 05: 48$

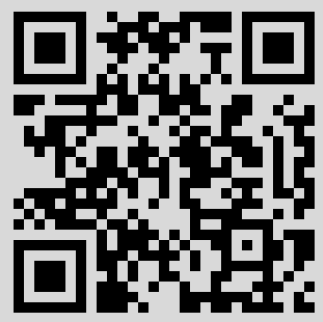




\title{
РЕКУРРЕНТНЫЕ СООТНОШЕНИЯ ДЛЯ $N=1$ СУПЕРСИММЕТРИЧНЫХ КОНФОРМНЫХ БЛОКОВ
}

\begin{abstract}
Приведены явные рекуррентные соотношения для четырехточечной функции суперконформных блоков, которые представляют собой частные вклады в четырехточечную корреляционную функцию, задаваемые данным конформным классом. Подход основан на аналитических свойствах суперконформных блоков как функций от конформных размерностей и центрального заряда суперконформной алгебры. Проведено сравнение результатов с явными аналитическими выражениями, полученными при специальных значениях параметров, отвечающих вырожденному операторному разложению. Эти рекуррентные соотношения представляют собой эффективный метод для численного исследования четырехточечной корреляционной функции в суперконформной теории поля в рамках бутстрапного подхода, подобно тому, что имеет место в случае чисто конформной симметрии.
\end{abstract}

Ключевые слова: $N=1$ суперконформная теория поля, четырехточечные функции конформных блоков, рекуррентные соотношения.

\section{1. ВВЕДЕНИЕ}

Прогресс, достигнутый в последнее время в полевой теории Лиувилля [1]-[3] и в двумерной квантовой гравитации Лиувилля [4]-[6], открывает возможности для новых приложений в струнной теории. Очень важным шагом в этом направлении оказываются суперсимметричные обобщения методов, используемых в бозонных теориях. Это особенно интересно, так как до сих пор не существует суперсимметричного обобщения техники матричных моделей, и поэтому подход, использующий суперсимметричную теорию Лиувилля, предложенный Поляковым [7], остается единственным перспективным методом. Одной из нерешенных проблем в этом подходе оказывается построение полного набора явных корреляционных функций минимальной супергравитации (минимальной суперструнной теории). Как и в бозонной конформной теории поля, основным методом вычислений в этой задаче является решение конформных уравнений бутстрапа. В этой программе важную роль играют функции конформных блоков [8]. К сожалению, замкнутые аналитические выражения для этих функций удается найти только при некоторых специальных значениях

${ }^{*}$ Институт теоретической и экспериментальной физики, Москва, Россия; International School for Advanced Studies (SISSA), Trieste, Italy. E-mail: belavinv@sissa.it 
конформных размерностей рассматриваемых полей. В этой статье найдены рекуррентные соотношения для четырехточечных функций конформных блоков в секторе Невье-Шварца $N=1$ суперсимметричной конформной теории поля, аналогичные соотношениям, найденным в работе [9] в бозонном случае. Последовательные итерации этих соотношений сходятся при достаточно малых $x$, что позволяет эффективно вычислить степенные разложения для корреляционных функций.

Структура статьи следующая. В разделе 2 кратко повторяются некоторые необходимые сведения из области $N=1$ суперсимметричной конформной теории поля (см. более детальное изложение в работах [10], [11]). В разделе 3 вводятся функции суперконформных блоков и описываются их аналитические свойства. В разделе 4 описаны сингулярности суперконформных блоков как функций от размерностей полей. В разделе 5 обсуждается асимптотическое поведение конформных блоков как функций от центрального заряда $c$ суперконформной алгебры и их сингулярности относительно с. После этого вводятся рекуррентные соотношения для функций суперконформных блоков. В разделе 6 обсуждается специальный вырожденный случай, в котором удается получить обыкновенные дифференциальные уравнения, которым удовлетворяют исследуемые функции суперконформных блоков, и проверить рекуррентные соотношения при соответствующем выборе параметров. Заключительные замечания представлены в разделе 7.

\section{2. $N=1$ СУПЕРКОНФОРМНАЯ ТЕОРИЯ ПОЛЯ}

Симметрия в суперконформной теории поля порождается голоморфными и антиголоморфными компонентами супертока $S$ и тензора энергии-импульса $T$. Будучи представлена через лорановские компоненты $S$ и $T$, алгебра принимает общепринятый вид алгебры Невье-Шварца-Рамона:

$$
\begin{aligned}
{\left[L_{n}, L_{m}\right] } & =(n-m) L_{n+m}+\frac{c}{8}\left(n^{3}-n\right) \delta_{n,-m}, \\
\left\{G_{r}, G_{s}\right\} & =2 L_{r+s}+\frac{1}{2} c\left(r^{2}-\frac{1}{4}\right) \delta_{r,-s}, \\
{\left[L_{n}, G_{r}\right] } & =\left(\frac{1}{2} n-r\right) G_{n+r},
\end{aligned}
$$

где $r, s \in \mathbb{Z}+1 / 2$ в секторе Невье-Шварца, $r, s \in \mathbb{Z}$ в секторе Рамона. Мы параметризуем центральный заряд как обычно в теории Лиувилля:

$$
c=1+2 Q^{2}, \quad Q=b+\frac{1}{b} .
$$

Локальные поля представляют собой представления со старшим весом для алгебры Невье-Шварца-Рамона. Каждое представление $\left[\Phi_{\Delta}\right]$ состоит из примарного поля конформной размерности $\Delta$ и всех его суперконформных потомков. Примарные суперполя Невье-Шварца ${ }^{1)}$ имеют вид

$$
\boldsymbol{\Phi}_{\Delta}(z)=\Phi_{\Delta}(x)+\theta \Psi_{\Delta}(x), \quad \Psi_{\Delta}(z)=G_{-\frac{1}{2}} \Phi_{\Delta}(z),
$$

\footnotetext{
1) Для простоты рассматривается только голоморфная составляющая.
} 
где $x$ и $\theta$ - голоморфные координаты на $(2+2)$-мерном суперпространстве (при этом через $\theta$ обозначена антикоммутирующая, "нечетная" координата). Введем также удобную параметризацию:

$$
\Delta(\lambda)=\frac{Q^{2}}{8}-\frac{\lambda^{2}}{2}
$$

Поле $\Phi_{m n}$, у которого индексы $m$ и $n$ - оба четные или оба нечетные положительные целые числа, отвечает "вырожденному" примарному полю в секторе Невье-Шварца, обладающему конформной размерностью $\Delta=\Delta\left(\lambda_{m n}\right)$ :

$$
\lambda_{m n}=\frac{m b^{-1}+n b}{2} .
$$

Общий вид оператора потомков в конформном классе $\left[\Phi_{\Delta}\right]$ следующий:

$$
\mathcal{L}_{\vec{k}}|\Delta\rangle=L_{-k_{1}} \ldots L_{-k_{n}} G_{-r_{1}} \ldots G_{-r_{m}} \Phi_{\Delta}
$$

где через $\vec{k}$ обозначен набор $\left\{k_{i}, r_{j}\right\}$, представляющий собой упорядоченное множество соответственно положительных целых и полуцелых чисел. Соотношение $\sum_{i} k_{i}+\sum_{j} r_{j}=N$ задает уровень модуля Верма, отвечающий суперконформному семейству $\left[\Phi_{\Delta}\right]$. Как обычно, тождества Уорда налагают ограничения на возможную зависимость корреляционных функций от координат. В частности, двухточечные функции полностью определены:

$$
\left\langle\boldsymbol{\Phi}_{1}\left(z_{1}\right) \boldsymbol{\Phi}_{2}\left(z_{2}\right)\right\rangle \sim \frac{\delta_{\Delta_{1}, \Delta_{2}}}{\left|z_{12}\right|^{4 \Delta_{1}}}
$$

где $z_{i k}=x_{i}-x_{k}+\theta_{i} \theta_{k}$. Трехточечная функция содержит зависимость от произвольной функции от "нечетного" суперпроективного инвариантного трехточечного соотношения:

$$
\theta_{123}=\frac{z_{23} \theta_{1}+z_{31} \theta_{2}+z_{12} \theta_{3}-\theta_{1} \theta_{2} \theta_{3}}{\left(z_{12} z_{13} z_{23}\right)^{1 / 2}},
$$

и может быть представлена в следующем виде:

$$
\left\langle\boldsymbol{\Phi}_{1}\left(z_{1}\right) \boldsymbol{\Phi}_{2}\left(z_{2}\right) \boldsymbol{\Phi}_{3}\left(z_{3}\right)\right\rangle=\frac{C_{123}+\left|\theta_{123}\right|^{2} \widetilde{C}_{123}}{\left|z_{12}\right|^{2\left(\Delta_{1}+\Delta_{2}-\Delta_{3}\right)}\left|z_{13}\right|^{2\left(\Delta_{1}+\Delta_{3}-\Delta_{2}\right)}\left|z_{23}\right|^{2\left(\Delta_{2}+\Delta_{3}-\Delta_{1}\right)}} .
$$

"Вырожденное" примарное поле $\Phi_{m n}$ содержит сингулярный вектор на уровне $N=$ $m n / 2$ [12]. Удобно ввести оператор рождения сингулярного вектора $D_{m n}[13]$, который, будучи примененным к $\Phi_{m n}$, порождает сингулярный вектор. Фиксируем нормировку, выбирая коэффициент при ведущем члене равным единице, $D_{m n}=$ $G_{-1 / 2}^{m n}+\cdots$. Первый нетривиальный нулевой вектор в секторе Невье-Шварца появляется на уровне $N=3 / 2$ :

$$
D_{13} \Phi_{13}=\left(L_{-1} G_{-\frac{1}{2}}+b^{2} G_{-\frac{3}{2}}\right) \Phi_{13}=0 .
$$




\section{3. ЧЕТЫРЕХТОЧЕЧНАЯ КОРРЕЛЯЦИОННАЯ ФУНКЦИЯ И КОНФОРМНЫЕ БЛОКИ}

Рассмотрим четырехточечную корреляционную функцию примарных суперполей в секторе Невье-Шварца $N=1$ суперсимметричной конформной теории. Для четырехточечной корреляционной функции имеются три независимых суперпроективных инварианта, один “четный” и два "нечетных" (см., например, [11]). Применяя суперконформную инвариантность (аналогично рассмотрению в работе [5]), можно записать

$$
\left\langle\boldsymbol{\Phi}_{1}\left(z_{1}\right) \boldsymbol{\Phi}_{2}\left(z_{2}\right) \boldsymbol{\Phi}_{3}\left(z_{3}\right) \boldsymbol{\Phi}_{4}\left(z_{4}\right)\right\rangle=\left|z_{34}\right|^{2 \gamma_{34}}\left|z_{13}\right|^{2 \gamma_{13}}\left|z_{23}\right|^{2 \gamma_{23}}\left|z_{12}\right|^{2 \gamma_{12}} g\left(z, \bar{z}, \tau_{1}, \bar{\tau}_{1}, \tau_{2}, \bar{\tau}_{2}\right),
$$

где

$$
\begin{aligned}
\gamma_{34} & =-2 \Delta_{4}, \\
\gamma_{13} & =-\Delta_{1}-\Delta_{3}+\Delta_{4}+\Delta_{2}, \\
\gamma_{23} & =-\Delta_{2}-\Delta_{3}+\Delta_{4}+\Delta_{1}, \\
\gamma_{12} & =-\Delta_{4}-\Delta_{1}-\Delta_{2}+\Delta_{3},
\end{aligned}
$$

и мы выбираем три независимых инварианта:

$$
z=\frac{z_{41} z_{23}}{z_{43} z_{21}}, \quad \tau_{1}=\theta_{213}, \quad \tau_{2}=-[z(z-1)]^{1 / 2} \theta_{214}
$$

Принимая в расчет суперпроективную инвариантность, можно, например, фиксировать

$$
\begin{gathered}
\theta_{1}=0, \quad \theta_{2}=0, \quad \theta_{3}=R \eta, \\
x_{1}=0, \quad x_{2}=1, \quad x_{3}=R, \quad x_{4}=x,
\end{gathered}
$$

где $R \rightarrow \infty$. Функция $g$ связана с корреляционной функцией бозонных компонент супермультиплета (3):

$$
\left\langle\Phi_{1}(0) \Phi_{2}(1) \Phi_{3}(\infty) \Phi_{4}(z)\right\rangle=g(z, \bar{z}, 0,0,0,0)=g_{0}(z, \bar{z}) .
$$

В дальнейшем ограничимся рассмотрением суперконформных блоков, дающих вклад в корреляционную функцию $g_{0}$. Обобщение на оставшиеся компоненты очевидно.

Аналогично случаю чисто конформной симметрии [8] можно выписать разложение в $s$-канале для корреляционной функции $g_{0}$ :

$$
\begin{aligned}
\left\langle\Phi_{1}(x) \Phi_{2}(0) \Phi_{3}(1) \Phi_{4}(\infty)\right\rangle=\sum_{\Delta} & {\left[C_{12}^{\Delta} C_{34}^{\Delta} F_{0}\left(\Delta, \Delta_{i}, x\right) F_{0}\left(\Delta, \Delta_{i}, \bar{x}\right)+\right.} \\
+ & \left.\widetilde{C}_{12}^{\Delta} \widetilde{C}_{34}^{\Delta} F_{1}\left(\Delta, \Delta_{i}, x\right) F_{1}\left(\Delta, \Delta_{i}, \bar{x}\right)\right] .
\end{aligned}
$$

Суперконформные блоки $F_{0}$ и $F_{1}$ определяются аналогично бозонному случаю (подробнее см., например, [14]):

$$
\begin{aligned}
& F_{0}\left(\Delta, \Delta_{i}, c, x\right)=x^{\Delta-\Delta_{1}-\Delta_{2}} \sum_{\substack{N \geqslant 0 \\
N \in \mathbb{Z}}} x^{N}{ }_{12}\langle N \mid N\rangle_{34}, \\
& F_{1}\left(\Delta, \Delta_{i}, c, x\right)=x^{\Delta-\Delta_{1}-\Delta_{2}} \sum_{\substack{N>0 \\
N \in \mathbb{Z}+1 / 2}} x^{N}{ }_{12}\langle N \mid N\rangle_{34},
\end{aligned}
$$


где вектор $|N\rangle$ представляет собой вклад потомков на $N$-м уровне, приходящий от промежуточного состояния с конформной размерностью $\Delta$, возникающего в операторном разложении $\Phi(x) \Phi(0)$ :

$$
\left[\Phi_{1}(x) \Phi_{2}(0)\right]_{\Delta}=x^{\Delta-\Delta_{1}-\Delta_{2}} \sum_{N=0}^{\infty} x^{N}|N\rangle_{12},
$$

где $|N\rangle_{12}=Q_{12}(N, \Delta)|\Delta\rangle, Q_{12}(N, \Delta)=\sum \beta_{12}(\vec{k}) \mathcal{L}_{\vec{k}}^{(N)}$, а $\mathcal{L}_{\vec{k}}$ определено в (6) (предполагается суммирование по всем потомкам на $N$-м уровне). Векторы $|N\rangle_{12}$ зависят не только от конформной размерности $\Delta$ и центрального заряда $c$, но также от размерностей полей $\Phi_{1}$ и $\Phi_{2}$, и поэтому мы присваиваем оператору $Q$ соответствующий индекс. Векторы $|N\rangle_{12}$, равно как и векторы $|\tilde{N}\rangle_{12}=\widetilde{Q}(N, \Delta)|\Delta\rangle$, появляющиеся в операторном разложении $\Psi_{1}(x) \Phi_{2}(0)$,

$$
\left[\Psi_{1}(x) \Phi_{2}(0)\right]_{\Delta}=x^{\Delta-\Delta_{1}-\Delta_{2}-1 / 2} \sum_{N=0}^{\infty} x^{N}|\widetilde{N}\rangle_{12}
$$

полностью задаются суперконформной симметрией. А именно, суперконформные связи приводят к соотношениям на векторы цепочки, растущей из вакуумного вектора $|\Delta\rangle$,

$$
\begin{gathered}
G_{k}|N\rangle_{12}=|\widetilde{N-k}\rangle_{12} \\
G_{k}|\tilde{N}\rangle_{12}=\left[\Delta+2 k \Delta_{1}-\Delta_{2}+N-k\right]|N-k\rangle_{12}
\end{gathered}
$$

при $k>0$. Из определения (17) и из свойств уравнений (20) можно получить, что начиная с уровня $m n / 2$ функции $F_{0}$ и $F_{1}$ имеют простые полюсы при $\Delta=\Delta_{m n}(c)$. Аналогично из этого соотношения следует, что конформные блоки, как функции центрального заряда $c$, имеют один простой полюс для каждой пары положительных целых чисел $m$ и $n(n>1)$ при $c=c_{m n}(\Delta)$, где

$$
\begin{gathered}
c_{m n}=5+2\left(T_{m n}+T_{m n}^{-1}\right), \\
T_{m n}=\frac{1-4 \Delta-m n+\sqrt{[(m n-1)+4 \Delta]^{2}-\left(m^{2}-1\right)\left(n^{2}-1\right)}}{n^{2}-1} .
\end{gathered}
$$

Вычеты функций $F_{0}$ и $F_{1}$ в этих полюсах пропорциональны функциям конформных блоков, отвечающих инвариантному подклассу, возникающему на $m n / 2$ уровне, на котором старший вектор представляет собой новое примарное поле с конформной размерностью $\Delta_{m n}=\Delta+m n / 2$ (см. аналогичное рассмотрение в работах [9] и [14]). Сингулярная часть этого выражения вкратце обсуждается в следующем разделе.

\section{4. СТРУКТУРА СИНГУЛЯРНОСТЕЙ ВЕКТОРОВ ЦЕПОЧКИ}

Сингулярная структура векторов цепочки будет рассмотрена более детально в последующей публикации. Здесь же будут представлены только основные результаты, 
касающиеся сингулярностей векторов цепочки, введенных в (20). При $\Delta \rightarrow \Delta_{m n}$

$$
\begin{aligned}
& \left|N=\frac{m n}{2}\right\rangle \rightarrow \frac{X_{m n}}{\Delta-\Delta_{m n}} D_{m n}|\Delta\rangle, \\
& \left|N=\frac{m n}{2}\right\rangle \rightarrow \frac{\bar{X}_{m n}}{\Delta-\Delta_{m n}} D_{m n}|\Delta\rangle .
\end{aligned}
$$

Чтобы получить зависимость коэффициентных функций $X_{m n}$ и $\bar{X}_{m n}$ от внешних размерностей, заметим, что при $\Delta=\Delta_{m n}(c)$ векторы цепочки все еще будут хорошо определены, если удовлетворяются некоторые правила “слияния" [8] между $\Delta$ и $\Delta_{i}$. Исследование правил “слияния”, основанное на анализе структурных функций [10], приводит к выражениям

$$
\begin{aligned}
& X_{m n}= \begin{cases}2^{-m n / 2 \frac{P_{m n}\left(\lambda_{1}+\lambda_{2}\right) P_{m n}\left(\lambda_{1}-\lambda_{2}\right)}{r_{m n}^{\prime}},} & m, n \text { четные, } \\
2^{(1-m n) / 2 \frac{P_{m n}\left(\lambda_{1}+\lambda_{2}\right) P_{m n}\left(\lambda_{1}-\lambda_{2}\right)}{r_{m n}^{\prime}},} & m, n \text { нечетные, }\end{cases} \\
& \bar{X}_{m n}= \begin{cases}2^{-m n / 2 \frac{\bar{P}_{m n}\left(\lambda_{1}+\lambda_{2}\right) \bar{P}_{m n}\left(\lambda_{1}-\lambda_{2}\right)}{r_{m n}^{\prime}},}, n \text { четные, } \\
2^{-(1+m n) / 2 \frac{\bar{P}_{m n}\left(\lambda_{1}+\lambda_{2}\right) \bar{P}_{m n}\left(\lambda_{1}-\lambda_{2}\right)}{r_{m n}^{\prime}},}\end{cases}
\end{aligned}
$$

Здесь

$$
P_{m n}=\prod_{(r, s) \in[m, n]}\left(\lambda-\lambda_{r s}\right)
$$

где

$$
[m, n]=\{1-m: 4: m-3,1-n: 4: n-3\} \cup\{3-m: 4: m-1,3-n: 4: n-1\},
$$

когда оба числа $m$ и $n$ четны, и

$$
[m, n]=\{3-m: 4: m-3,1-n: 4: n-1\} \cup\{1-m: 4: m-1,3-n: 4: n-3\},
$$

когда оба числа $m$ и $n$ нечетны;

$$
\bar{P}_{m n}=\prod_{(r, s) \in \overline{[m, n]}}\left(\lambda-\lambda_{r s}\right),
$$

где

$$
\overline{[m, n]}=\{1-m: 4: m-3,3-n: 4: n-1\} \cup\{3-m: 4: m-1,1-n: 4: n-3\},
$$

когда оба числа $m$ и $n$ четны, и

$$
\overline{[m, n]}=\{3-m: 4: m-3,3-n: 4: n-3\} \cup\{1-m: 4: m-1,1-n: 4: n-1\},
$$

когда оба числа $m$ и $n$ нечетны. Простой анализ соотношений (20) приводит к выводу, что множитель $r_{m n}^{\prime}$ зависит только от $c$ и $\Delta$. В последующей публикации будет прояснено, что этот коэффициент связан с нормой соответствующего сингулярного вектора,

$$
\| D_{m n}|\Delta\rangle \|^{2}=r_{m n}^{\prime}\left(\Delta-\Delta_{m n}\right)
$$

3 Теоретическая и математическая физика, т. 152, № 3, 2007 г. 
при $\Delta \rightarrow \Delta_{m n}$. Принимая в расчет результаты работы [13], запишем

$$
r_{m n}^{\prime}=\frac{r_{m n}}{\lambda_{m n}}
$$

где

$$
r_{m n}=2^{1-m n} \prod_{(k, l) \in[m, n]}\left(k b^{-1}+l b\right),
$$

$[m, n]=\{1-m: 2: m-1,1-n: 2: n-1\} \cup\{2-m: 2: m, 2-n: 2: n\} \backslash(0,0)$.

Выражения вида $a: d: b$ (“от $a$ до $b$ с шагом $d$ ") в формулах выше обозначают наборы чисел $a, a+d, a+2 d, \ldots, b$. Через $\{A, B\}$ обозначено множество пар $(k, l)$, в которых $k$ и $l$ независимо пробегают множества $A$ и $B$, а $\left\{A_{1}, B_{1}\right\} \cup\left\{A_{2}, M_{2}\right\}$ обозначает стандартное объединение двух множеств. Наконец, “. . \ $\backslash(0,0)$ ” означает исключение пары индексов $(0,0)$ из набора.

Таким же образом векторы цепочки при $N>m n / 2$ также обладают простыми полюсами при $\Delta=\Delta_{m n}$, и, следовательно,

$$
\begin{gathered}
\left|N \geqslant \frac{m n}{2}\right\rangle \rightarrow\left\{\begin{array}{lll}
\frac{X_{m n}}{\Delta-_{m n}} Q\left(N-\frac{m n}{2}, \Delta_{m n}+\frac{m n}{2}\right) D_{m n}|\Delta\rangle, & N-\frac{m n}{2} & \text { целое, } \\
\frac{\bar{X}_{m n}}{\Delta-\Delta_{m n}} Q\left(N-\frac{m n}{2}, \Delta_{m n}+\frac{m n}{2}\right) D_{m n}|\Delta\rangle, & N-\frac{m n}{2} & \text { полуцелое, }
\end{array}\right. \\
\left|N \geqslant \frac{m n}{2}\right\rangle \rightarrow\left\{\begin{array}{lll}
\frac{\bar{X}_{m n}}{\Delta-\Delta_{m n}} \widetilde{Q}\left(N-\frac{m n}{2}, \Delta_{m n}+\frac{m n}{2}\right) D_{m n}|\Delta\rangle, & N-\frac{m n}{2} & \text { целое, } \\
\frac{X_{m n}}{\Delta-\Delta_{m n}} \widetilde{Q}\left(N-\frac{m n}{2}, \Delta_{m n}+\frac{m n}{2}\right) D_{m n}|\Delta\rangle, & N-\frac{m n}{2} & \text { полуцелое. }
\end{array}\right.
\end{gathered}
$$

\section{5. РЕКУРРЕНТНЫЕ СООТНОШЕНИЯ}

Рассмотрение в предыдущем разделе приводит к соотношениям на конформные блоки, рассматриваемые как функции внутренней конформной размерности $\Delta$ :

$$
\begin{aligned}
& F_{0}\left(\Delta \rightarrow \Delta_{m n}, \Delta_{i}, c, x\right)=\left\{\begin{array}{lll}
\frac{R_{m n}}{\Delta \bar{R}_{m n}} F_{0}\left(\Delta_{m,-n}, \Delta_{i}, c, x\right), & m, n & \text { четные, } \\
\frac{\bar{R}_{m n}}{\Delta-\Delta_{m n}} F_{1}\left(\Delta_{m,-n}, \Delta_{i}, c, x\right), & m, n & \text { нечетные, }
\end{array}\right. \\
& F_{1}\left(\Delta \rightarrow \Delta_{m n}, \Delta_{i}, c, x\right)=\left\{\begin{array}{lll}
\frac{\bar{R}_{m n}}{\Delta-\Delta_{m n}} F_{1}\left(\Delta_{m,-n}, \Delta_{i}, c, x\right), & m, n & \text { четные }, \\
\frac{R_{m n}}{\Delta-\Delta_{m n}} F_{0}\left(\Delta_{m,-n}, \Delta_{i}, c, x\right), & m, n & \text { нечетные },
\end{array}\right.
\end{aligned}
$$

где

$$
R_{m n}=X_{m n}^{(12)} X_{m n}^{(34)} r_{m n}^{\prime}, \quad \bar{R}_{m n}=\bar{X}_{m n}^{(12)} \bar{X}_{m n}^{(34)} r_{m n}^{\prime}
$$

Тем самым вычеты в полюсах конформных блоков, рассматриваемых как функции центрального заряда $c$, также полностью определены:

$$
\begin{aligned}
& F_{0}\left(\Delta, \Delta_{i}, c \rightarrow c_{m n}, x\right)=\left\{\begin{array}{lll}
\frac{R_{m n}^{\prime}}{{ }^{c} c_{m n}} F_{0}\left(\Delta+\frac{m n}{2}, \Delta_{i}, c_{m n}, x\right), & m, n & \text { четные, } \\
\frac{\bar{R}_{m n}^{\prime}}{c-c_{m n}} F_{1}\left(\Delta+\frac{m n}{2}, \Delta_{i}, c_{m n}, x\right), & m, n & \text { нечетные, }
\end{array}\right. \\
& F_{1}\left(\Delta, \Delta_{i}, c \rightarrow c_{m n}, x\right)=\left\{\begin{array}{lll}
\frac{\bar{R}_{m n}^{\prime}}{c-c_{m n}} F_{1}\left(\Delta+\frac{m n}{2}, \Delta_{i}, c_{m n}, x\right), & m, n & \text { четные, } \\
\frac{R_{m n}^{\prime}}{c-c_{m n}} F_{0}\left(\Delta+\frac{m n}{2}, \Delta_{i}, c_{m n}, x\right), & m, n & \text { нечетные, }
\end{array}\right.
\end{aligned}
$$


где $c_{m n}$ задано в $(21)$, а коэффициенты $R_{m n}$ и $R_{m n}^{\prime}$ по существу совпадают и отличаются только заменой переменной:

$$
\begin{gathered}
R_{m n}^{\prime}=R_{m n}\left(c_{m n}\right)\left(\frac{\partial \Delta_{m n}}{\partial c}\right)^{-1} \\
\frac{\partial \Delta_{m n}}{\partial c}=\frac{1}{16} \frac{\left(n^{2}-1\right) T_{m n}-\left(m^{2}-1\right) T_{m n}^{-1}}{T_{m n}-T_{m n}^{-1}} .
\end{gathered}
$$

При $c=\infty$ соотношения (20) упрощаются и могут быть решены явно. Это приводит к выражениям для асимптотических значений $F_{0}$ и $F_{1}$ в терминах гипергеометрических функций:

$$
\begin{gathered}
F_{0}(c \rightarrow \infty)=f_{0}\left(\Delta, \Delta_{i}, x\right)=x^{\Delta-\Delta_{1}-\Delta_{2}}{ }_{2} F_{1}\left(\Delta+\Delta_{1}-\Delta_{2}, \Delta+\Delta_{3}-\Delta_{4}, 2 \Delta, x\right), \\
F_{1}(c \rightarrow \infty)=f_{1}\left(\Delta, \Delta_{i}, x\right)=\frac{1}{2 \Delta} x^{\Delta-\Delta_{1}-\Delta_{2}+1 / 2} \times \\
\quad \times{ }_{2} F_{1}\left(\Delta+\Delta_{1}-\Delta_{2}+1 / 2, \Delta+\Delta_{3}-\Delta_{4}+1 / 2,2 \Delta+1, x\right) .
\end{gathered}
$$

Таким образом, ясно, что можно выписать следующие соотношения для конформных блоков $F_{0}$ и $F_{1}$ :

$$
\begin{gathered}
F_{0}\left(\Delta, \Delta_{i}, c, x\right)=f_{0}\left(\Delta, \Delta_{i}, x\right)+\sum_{\substack{\{m, n\} \\
m, n \text { четные }}} \frac{R_{m n}^{\prime}}{c-c_{m n}} F_{0}\left(\Delta+\frac{m n}{2}, \Delta_{i}, c_{m n}, x\right)+ \\
+\sum_{\substack{\{m, n\} \\
m, n \text { нечетные }, n>1}} \frac{\bar{R}_{m n}^{\prime}}{c-c_{m n}} F_{1}\left(\Delta+\frac{m n}{2}, \Delta_{i}, c_{m n}, x\right), \\
F_{1}\left(\Delta, \Delta_{i}, c, x\right)=f_{1}\left(\Delta, \Delta_{i}, x\right)+\sum_{\substack{\{m, n\} \\
m, n \text { четные }}} \frac{\bar{R}_{m n}^{\prime}}{c-c_{m n}} F_{1}\left(\Delta+\frac{m n}{2}, \Delta_{i}, c_{m n}, x\right)+ \\
+\sum_{\substack{\{m, n\} \\
m, n \text { нечетные }, n>1}} \frac{R_{m n}^{\prime}}{c-c_{m n}} F_{0}\left(\Delta+\frac{m n}{2}, \Delta_{i}, c_{m n}, x\right) .
\end{gathered}
$$

Можно разложить выражения для $F_{0}$ и $F_{1}$ в ряды по $x$, итерируя уравнения $(29)$ :

$$
\begin{aligned}
& F_{0}\left(\Delta, \Delta_{i}, c, x\right)=x^{\Delta-\Delta_{1}-\Delta_{2}} \sum_{k=0}^{\infty} F_{0}^{(k)} x^{k}, \\
& F_{1}\left(\Delta, \Delta_{i}, c, x\right)=x^{\Delta-\Delta_{1}-\Delta_{2}+1 / 2} \sum_{k=0}^{\infty} F_{1}^{(k)} x^{k} .
\end{aligned}
$$

Для краткости в дальнейшем опустим зависимость от внешних размерностей, которая ниже всюду одна и та же. Применяя рекуррентные соотношения (29), можно легко найти первые члены разложения:

$$
F_{0}^{(0)}=f_{0}^{(0)}(\Delta)
$$




$$
\begin{gathered}
F_{0}^{(1)}=f_{0}^{(1)}(\Delta), \\
F_{0}^{(2)}=f_{0}^{(2)}(\Delta)+\frac{\bar{R}_{13}^{\prime}}{c-c_{13}} f_{1}^{(0)}\left(\Delta+\frac{3}{2}\right)+\frac{R_{22}^{\prime}}{c-c_{22}} f_{0}^{(0)}(\Delta+2), \\
F_{1}^{(0)}=f_{1}^{(0)}(\Delta), \\
F_{1}^{(1)}=f_{1}^{(1)}(\Delta)+\frac{\bar{R}_{13}^{\prime}}{c-c_{13}} f_{0}^{(0)}\left(\Delta+\frac{3}{2}\right) \\
F_{1}^{(2)}=f_{1}^{(2)}(\Delta)+\frac{R_{13}^{\prime}}{c-c_{13}} f_{0}^{(1)}\left(\Delta+\frac{3}{2}\right)+\frac{\bar{R}_{22}^{\prime}}{c-c_{22}} f_{1}^{(0)}(\Delta+2)+\frac{R_{15}^{\prime}}{c-c_{15}} f_{0}^{(0)}\left(\Delta+\frac{5}{2}\right) .
\end{gathered}
$$

Используя формулы (27) вместе с выражениями, полученными в предыдущем разделе, можно выписать явно первые коэффициенты:

$$
\begin{aligned}
& F_{0}^{(0)}=1, \\
& F_{0}^{(1)}=\left(\Delta+\Delta_{1}-\Delta_{2}\right)\left(\Delta+\Delta_{3}-\Delta_{4}\right)(2 \Delta)^{-1} \text {, } \\
& F_{0}^{(2)}=\left(\Delta+\Delta_{1}-\Delta_{2}\right)\left(1+\Delta+\Delta_{1}-\Delta_{2}\right)\left(\Delta+\Delta_{3}-\Delta_{4}\right)\left(1+\Delta+\Delta_{3}-\Delta_{4}\right) \times \\
& \times[4 \Delta(1+2 \Delta)]^{-1}+\left[\Delta^{2}-3\left(\Delta_{1}-\Delta_{2}\right)^{2}+2 \Delta\left(\Delta_{1}+\Delta_{2}\right)\right] \times \\
& \times\left[\Delta^{2}-3\left(\Delta_{3}-\Delta_{4}\right)^{2}+2 \Delta\left(\Delta_{3}+\Delta_{4}\right)\right] \times \\
& \times[2 \Delta(3+2 \Delta)(-3+3 c+16 \Delta)]^{-1}+ \\
& +\left[\Delta_{1}-2\left(\Delta_{1}-\Delta_{2}\right)^{2}+\Delta_{2}+\Delta\left(-1+2 \Delta_{1}+2 \Delta_{2}\right)\right] \times \\
& \times\left[\Delta_{3}-2\left(\Delta_{3}-\Delta_{4}\right)^{2}+\Delta_{4}+\Delta\left(-1+2 \Delta_{3}+2 \Delta_{4}\right)\right] \times \\
& \times\left\{\left[c+2(-3+c) \Delta+4 \Delta^{2}\right][3+4 \Delta(2+\Delta)]\right\}^{-1}, \\
& F_{1}^{(0)}=(2 \Delta)^{-1} \text {, } \\
& F_{1}^{(1)}=\left(1+2 \Delta+2 \Delta_{1}-2 \Delta_{2}\right)\left(1+2 \Delta+2 \Delta_{3}-2 \Delta_{4}\right)[8 \Delta(1+2 \Delta)]^{-1}, \\
& F_{1}^{(2)}=128^{-1}\left(1+2 \Delta+2 \Delta_{1}-2 \Delta_{2}\right)\left(3+2 \Delta+2 \Delta_{1}-2 \Delta_{2}\right) \times \\
& \times\left(1+2 \Delta+2 \Delta_{3}-2 \Delta_{4}\right)\left(3+2 \Delta+2 \Delta_{3}-2 \Delta_{4}\right) \times \\
& \times\left[\Delta\left(1+3 \Delta+2 \Delta^{2}\right)\right]^{-1}+ \\
& +\left(3+2 \Delta+2 \Delta_{1}-2 \Delta_{2}\right)\left(\Delta_{1}-\Delta_{2}\right)\left(3+2 \Delta+2 \Delta_{3}-2 \Delta_{4}\right) \times \\
& \times\left(\Delta_{3}-\Delta_{4}\right)\left\{\left[c+2(-3+c) \Delta+4 \Delta^{2}\right][3+4 \Delta(2+\Delta)]\right\}^{-1}+ \\
& +\left\{1+4 \Delta_{1}+4 \Delta_{2}+2\left[-6\left(\Delta_{1}-\Delta_{2}\right)^{2}+\Delta\left(-1+2 \Delta_{1}+2 \Delta_{2}\right)\right]\right\} \times \\
& \times\left(\Delta_{1}, \Delta_{2} \rightarrow \Delta_{3}, \Delta_{4}\right) \times \\
& \times\left\{64 \Delta(1+\Delta)(2+\Delta)\left[5-11 \Delta+2 \Delta^{2}+3 c(1+\Delta)\right]\right\}^{-1}+ \\
& +\left\{3\left(-1+4 \Delta_{1}+4 \Delta_{2}\right)+4\left[\Delta^{2}-3\left(\Delta_{1}-\Delta_{2}\right)^{2}+\right.\right. \\
& \left.\left.+\Delta\left(1+2 \Delta_{1}+2 \Delta_{2}\right)\right]\right\} \times \\
& \times\left(\Delta_{1}, \Delta_{2} \rightarrow \Delta_{3}, \Delta_{4}\right)[64 \Delta(2+\Delta)(3+2 \Delta)(-3+3 c+16 \Delta)]^{-1} \text {. }
\end{aligned}
$$




\section{6. ДИФФЕРЕНЦИАЛЬНЫЕ УРАВНЕНИЯ, ОТВЕЧАЮЩИЕ НУЛЕВОМУ ВЕКТОРУ $(1,3)$}

Поскольку сингулярный вектор (10) обращается в нуль, выполнено дифференциальное уравнение

$$
\left\{-b^{-2} \partial_{4} D_{4}+\sum_{i=1}^{3}\left\{\frac{2 \Delta_{i}}{z_{4 i}^{2}} \theta_{4 i}+\frac{1}{z_{4 i}}\left[2 \theta_{4 i} \partial_{i}-D_{i}\right]\right\}\right\}\left\langle\boldsymbol{\Phi}_{1}\left(z_{1}\right) \boldsymbol{\Phi}_{2}\left(z_{2}\right) \boldsymbol{\Phi}_{3}\left(z_{3}\right) \boldsymbol{\Phi}_{13}\left(z_{4}\right)\right\rangle=0
$$

где $D=\partial_{\theta}-\theta \partial_{x}$. С учетом (11) это уравнение сведется к дифференциальному уравнению на $g\left(z, \bar{z}, \tau_{1}, \bar{\tau}_{1}, \tau_{2}, \bar{\tau}_{2}\right)$ :

$$
\begin{gathered}
{\left[-b^{-2} \partial_{4} D_{4}-b^{-2} \frac{\gamma_{34}}{z_{34}}\left(\theta_{43} \partial_{4}-D_{4}\right)+\sum_{i=1}^{3}\left[\frac{2 \Delta_{i}}{z_{4 k}^{2}} \theta_{4 i}+\frac{1}{z_{4 i}}\left(2 \theta_{4 i} \partial_{i}-D_{i}\right)\right]+\right.} \\
\left.+\frac{\gamma_{12}\left(\theta_{41}+\theta_{42}\right)}{z_{41} z_{42}}+\frac{\gamma_{13}\left(\theta_{41}+\theta_{43}\right)}{z_{41} z_{43}}+\frac{\gamma_{23}\left(\theta_{42}+\theta_{43}\right)}{z_{42} z_{43}}+\frac{\gamma_{34} \theta_{43}}{z_{43}^{2}}\right] g=0 .
\end{gathered}
$$

В соответствии с (15) представим $g=g_{0}(z)+g_{1}(z) \tau_{1}+g_{2}(z) \tau_{2}+g_{3}(z) \tau_{1} \tau_{2}$ (также не забывая про антиголоморфную зависимость). Уравнение (39) расщепляется на две независимых системы обыкновенных дифференциальных уравнений для $g_{i}$. Для целей данной работы выпишем явно систему для $g_{0}$ и $g_{3}$ :

$$
\begin{array}{r}
-b^{-2} z g_{0}^{\prime \prime}+\frac{3 z-2}{z-1} g_{0}^{\prime}+b^{-2} g_{3}^{\prime}+\left[\frac{\gamma_{13}}{z}+\frac{\gamma_{23}}{z-1}\right] g_{0}+\frac{1-2 z}{z(z-1)} g_{3}=0 \\
b^{-2} g_{0}^{\prime \prime}+\frac{1-3 z}{z(z-1)} g_{0}^{\prime}+\left[\frac{2 \Delta_{1}}{z^{2}}+\frac{2 \Delta_{2}}{(z-1)^{2}}+\frac{2 \gamma_{12}}{z(z-1)}\right] g_{0}+\frac{1}{z(z-1)} g_{3}=0
\end{array}
$$

Три независимых решения для $g_{0}$ с диагональной монодромией в окрестности точки $x=0$ представляют собой конформные блоки в $s$-канале при специальном выборе параметров $\Delta_{1}=\Delta_{13}, \Delta_{2}=\Delta\left(\lambda_{1}\right), \Delta_{3}=\Delta\left(\lambda_{2}\right), \Delta_{4}=\Delta\left(\lambda_{3}\right)$ и $\Delta=\Delta^{( \pm)}=$ $\Delta\left(\lambda_{1} \pm b\right)$ или $\Delta=\Delta^{(0)}=\Delta\left(\lambda_{1}\right)$, отвечающем общей нормировке $A_{0}^{( \pm)}=1, A_{0}^{(0)}=$ $1 /\left(2 \Delta\left(\lambda_{1}\right)\right)$ :

$$
\begin{aligned}
& g_{0}^{( \pm)}=x^{\Delta-\Delta_{1}-\Delta_{2}} \sum_{n=0}^{\infty} A_{n}^{( \pm)} x^{n}=F_{0}\left(\Delta^{( \pm)}, \Delta_{i}, c, x\right), \\
& g_{0}^{(0)}=x^{\Delta-\Delta_{1}-\Delta_{2}+1 / 2} \sum_{n=0}^{\infty} A_{n}^{(0)} x^{n}=F_{1}\left(\Delta^{(0)}, \Delta_{i}, c, x\right) .
\end{aligned}
$$

Первые члены разложения в ряд можно легко найти, подставляя эти разложения в дифференциальные уравнения (40) и разрешая рекуррентные соотношения на коэффициенты порядок за порядком (что было сделано с помощью программы Matematika):

$$
\begin{gathered}
A_{0}^{(+)}=1, \\
A_{1}^{(+)}=\left(1+2 b^{2}-3 b^{4}-8 b^{3} \lambda_{1}-4 b^{2} \lambda_{1}^{2}-4 b^{2} \lambda_{2}^{2}+4 b^{2} \lambda_{3}^{2}\right)\left[4\left(-1+b^{2}+2 b \lambda_{1}\right)\right]^{-1},
\end{gathered}
$$




$$
\begin{aligned}
& A_{2}^{(+)}=\left(21+4 b^{2}-90 b^{4}+84 b^{6}-19 b^{8}-62 b \lambda_{1}-152 b^{3} \lambda_{1}+380 b^{5} \lambda_{1}-184 b^{7} \lambda_{1}+\right. \\
& +18 b^{9} \lambda_{1}+8 b^{2} \lambda_{1}^{2}+432 b^{4} \lambda_{1}^{2}-472 b^{6} \lambda_{1}^{2}+96 b^{8} \lambda_{1}^{2}+112 b^{3} \lambda_{1}^{3}-416 b^{5} \lambda_{1}^{3}+ \\
& +176 b^{7} \lambda_{1}^{3}-112 b^{4} \lambda_{1}^{4}+128 b^{6} \lambda_{1}^{4}+32 b^{5} \lambda_{1}^{5}-72 b^{2} \lambda_{2}^{2}+176 b^{4} \lambda_{2}^{2}-40 b^{6} \lambda_{2}^{2}+ \\
& +240 b^{3} \lambda_{1} \lambda_{2}^{2}-288 b^{5} \lambda_{1} \lambda_{2}^{2}+48 b^{7} \lambda_{1} \lambda_{2}^{2}-224 b^{4} \lambda_{1}^{2} \lambda_{2}^{2}+128 b^{6} \lambda_{1}^{2} \lambda_{2}^{2}+64 b^{5} \lambda_{1}^{3} \lambda_{2}^{2}- \\
& -48 b^{4} \lambda_{2}^{4}+32 b^{5} \lambda_{1} \lambda_{2}^{4}+24 b^{2} \lambda_{3}^{2}-144 b^{4} \lambda_{3}^{2}+56 b^{6} \lambda_{3}^{2}-112 b^{3} \lambda_{1} \lambda_{3}^{2}+288 b^{5} \lambda_{1} \lambda_{3}^{2}- \\
& -48 b^{7} \lambda_{1} \lambda_{3}^{2}+160 b^{4} \lambda_{1}^{2} \lambda_{3}^{2}-128 b^{6} \lambda_{1}^{2} \lambda_{3}^{2}-64 b^{5} \lambda_{1}^{3} \lambda_{3}^{2}+96 b^{4} \lambda_{2}^{2} \lambda_{3}^{2}-64 b^{5} \lambda_{1} \lambda_{2}^{2} \lambda_{3}^{2}- \\
& \left.-48 b^{4} \lambda_{3}^{4}+32 b^{5} \lambda_{1} \lambda_{3}^{4}\right)\left[64\left(-1+b \lambda_{1}\right)\left(-3+b^{2}+2 b \lambda_{1}\right)\left(-1+b^{2}+2 b \lambda_{1}\right)\right]^{-1} \text {, } \\
& A_{0}^{(0)}=4 b^{2}\left[\left(1+b^{2}-2 b \lambda_{1}\right)\left(1+b^{2}+2 b \lambda_{1}\right)\right]^{-1}, \\
& A_{1}^{(0)}=\left[-2 b^{4}\left(9+6 b^{2}+b^{4}-4 b^{2} \lambda_{1}^{2}-8 \lambda_{2}^{2}-4 b^{2} \lambda_{2}^{2}+8 \lambda_{3}^{2}+4 b^{2} \lambda_{3}^{2}\right)\right] \times \\
& \times\left[\left(1+b^{2}-2 b \lambda_{1}\right)\left(3+b^{2}-2 b \lambda_{1}\right)\left(1+b^{2}+2 b \lambda_{1}\right)\left(3+b^{2}+2 b \lambda_{1}\right)\right]^{-1}, \\
& A_{2}^{(0)}=\left[b ^ { 2 } \left(-72-534 b^{2}-287 b^{4}+48 b^{6}+62 b^{8}+14 b^{10}+b^{12}+32 b^{2} \lambda_{1}^{2}+288 b^{4} \lambda_{1}^{2}-\right.\right. \\
& -8 b^{6} \lambda_{1}^{2}-48 b^{8} \lambda_{1}^{2}-8 b^{10} \lambda_{1}^{2}-32 b^{6} \lambda_{1}^{4}+16 b^{8} \lambda_{1}^{4}+544 b^{2} \lambda_{2}^{2}+128 b^{4} \lambda_{2}^{2}- \\
& -200 b^{6} \lambda_{2}^{2}-80 b^{8} \lambda_{2}^{2}-8 b^{10} \lambda_{2}^{2}-128 b^{4} \lambda_{1}^{2} \lambda_{2}^{2}+32 b^{8} \lambda_{1}^{2} \lambda_{2}^{2}+128 b^{4} \lambda_{2}^{4}+96 b^{6} \lambda_{2}^{4}+ \\
& +16 b^{8} \lambda_{2}^{4}-256 b^{2} \lambda_{3}^{2}+208 b^{4} \lambda_{3}^{2}+328 b^{6} \lambda_{3}^{2}+96 b^{8} \lambda_{3}^{2}+8 b^{10} \lambda_{3}^{2}-64 b^{6} \lambda_{1}^{2} \lambda_{3}^{2}- \\
& -32 b^{8} \lambda_{1}^{2} \lambda_{3}^{2}-256 b^{4} \lambda_{2}^{2} \lambda_{3}^{2}-192 b^{6} \lambda_{2}^{2} \lambda_{3}^{2}-32 b^{8} \lambda_{2}^{2} \lambda_{3}^{2}+128 b^{4} \lambda_{3}^{4}+96 b^{6} \lambda_{3}^{4}+ \\
& \left.\left.+16 b^{8} \lambda_{3}^{4}\right)\right]\left[2\left(1+b^{2}-2 b \lambda_{1}\right)\left(3+b^{2}-2 b \lambda_{1}\right)\left(5+b^{2}-2 b \lambda_{1}\right) \times\right. \\
& \left.\times\left(1+b^{2}+2 b \lambda_{1}\right)\left(3+b^{2}+2 b \lambda_{1}\right)\left(5+b^{2}+2 b \lambda_{1}\right)\right]^{-1} \text {. }
\end{aligned}
$$

Эти выражения совпадают с выражениями для соответствующих коэффициентов (32)-(34) и (35)-(37), вычисленными при тех же значениях параметров, что подтверждает справедливость рекуррентных соотношений (29).

\section{7. ЗАКЛЮЧЕНИЕ}

Рекуррентные соотношения (29) для функций суперконформных блоков и их проверка в разделе 6 представляют собой основной результат данной работы. С помощью этих соотношений можно эффективно вычислять суперконформные блоки при произвольных значениях параметров. Они подходят также и для численных вычислений четырехточечных корреляционных функций как для примарных полей общего вида в секторе Невье-Шварца, так и для вырожденных примарных полей в частности. Заметим, что для четырехточечных конформных блоков (а также для суперконформных блоков) имеет место [9], [15] так называемое $q$-представление рекуррентных соотношений, которое оказывается предпочтительным, так как оно сходится всюду в комплексной плоскости с тремя выколотыми точками.

Представления вида (16) можно выписать также и для высших многоточечных корреляционных функций. Они включают в себя многоточечные конформные блоки, которые имеют структуру, гораздо более сложную, чем четырехточечные блоки. Мы надеемся, что подход, предложенный в этой статье, окажется плодотворным при решении чрезвычайно интересной задачи обобщения рекуррентных соотношений на 
высшие многоточечные конформные блоки и, в частности, при исследовании вопроса о соответствующих обобщенных $q$-представлениях.

Благодарности. Автор признателен А. А. Белавину и Ал. Б. Замолодчикову за полезные обсуждения и за возможность использовать наши совместные результаты до их опубликования. Автор также благодарен Дж. Дельфино и Дж. Муссардо за поддержку и интерес к его работе. Автор благодарен SISSA (Триест, Италия) за гостеприимство в период выполнения работы и INFN за финансовую поддержку.

\section{Список литературы}

[1] A. B. Zamolodchikov, Al. B. Zamolodchikov, Nucl. Phys. B, 477 (1996), 577-605; Structure constants and conformal bootstrap in Liouville field theory, hep-th/9506136.

[2] H. Dorn, H.-J. Otto, Nucl. Phys. B, 429 (1994), 375-388; hep-th/9403141.

[3] J. Teschner, Class. Quantum Grav., 18 (2001), R153-R222; hep-th/0104158.

[4] Al. Zamolodchikov, Internat. J. Modern Phys. A, 19:suppl2 (2004), 510-523; hep-th/0312279.

[5] Ал. Б. Замолодчиков, ТМФ, 142 (2005), 218-234; hep-th/0505063.

[6] А. А. Белавин, Ал. Б. Замолодчиков, ТМФ, 147 (2006), 339-371.

[7] A. M. Polyakov, Phys. Lett. B, 103 (1981), 211-213.

[8] A. A. Belavin, A. M. Polyakov, A. B. Zamolodchikov, Nucl. Phys. B, 241 (1984), 333-380.

[9] Al. Zamolodchikov, Commun. Math. Phys., 96 (1984), 419-422.

[10] Ал. Б. Замолодчиков, Р. Погосян, ЯФ, 47 (1988), 1461-1474.

[11] L. Alvarez-Gaumé, Ph. Zaugg, Ann. Phys., 215 (1992), 171-230; hep-th/9109050.

[12] В. Г. Кац, Бесконечномерные алгебры Ли, Мир, М., 1993.

[13] А.А. Белавин, Ал.Б. Замолодчиков, Писъма в ЖЖЭТФ, 84 (2006), 496-502; hep-th/0610316.

[14] A.B. Zamolodchikov, Al. B. Zamolodchikov, Conformal field theory and 2-D critical phenomena. Part III. Conformal bootstrap and degenerate representations of conformal algebra, ITEP-90-31, 1990.

[15] Ал. Б. Замолодчиков, ТМФ, 73 (1987), 103-110. 